\title{
Comportamento sedentário: conceito, implicações fisiológicas e os procedimentos de avaliação
}

\author{
Sedentary behavior: concept, physiological implications and the assess- \\ ment procedures
}

\author{
Joilson Meneguci ${ }^{*}$, Douglas Assis Teles Santos², Rodrigo Barboza Silva1, Rafaela Go- \\ mes Santos ${ }^{1}$, Jeffer Eidi Sasaki ${ }^{1}$, Sheilla Tribess ${ }^{1}$, Renata Damião ${ }^{1}$, Jair Sindra Virtuoso \\ Júnior $^{1}$
}

\begin{abstract}
O presente estudo de revisão narrativa teve como objetivos: a) rever os termos operacionais utilizados para a caracterização do comportamento sedentário; b) descrever as implicações para a saúde; e c) os principais métodos de avaliação do comportamento sedentário. Comportamento sedentário é o termo direcionado para as atividades que são realizadas na posição deitada ou sentada e que não aumentam o dispêndio energético acima dos níveis de repouso, $\leq 1.5$ equivalentes metabólicos (METs). Ao analisar as implicações fisiológicas destes comportamentos para a saúde das pessoas é possível destacar a redução e/ou a cessação da contratilidade muscular como desencadeador do processo de diminuição da utilização da glicose pelos músculos, do aumento da insulina e do favorecimento da produção de lipídios que serão preferencialmente armazenados no tecido adiposo da região central do corpo, o qual por sua vez produz moléculas inflamatórias precursoras das doenças crónicas não transmissíveis. A inatividade física e a exposição prolongada a comportamentos sedentários devem ser avaliadas distintamente, respeitando as bases teóricas de cada constructo. O efeito prolongado da exposição aos comportamentos sedentários e da inatividade física ao longo do curso da vida favorece a potencialização dos efeitos nocivos de tais comportamentos na velhice e a mortalidade precoce.
\end{abstract}

Palavras-chaves: atividade física, estilo de vida sedentário, revisão de literatura.

ABSTRACT

This present study aimed to: a) review the operational terms of sedentary behaviour characterization, b) describe the health implications, and c) sedentary behaviour main evaluation methods. Sedentary behaviour is the word targeting activities that are performed in lying or sitting position and that do not increase energy expenditure above resting levels, $\leq 1.5$ metabolic equivalent units (METs). Through the analysis of the physiological implications of such behaviour to people's health it is possible to highlight the reduction and / or cessation of muscle contractility as a beginner of the reducing process of the use of glucose by muscles, increasing insulin and facilitating the production of lipids that are preferentially stored in the central body adipose tissue, which in turn produces inflammatory molecules which are the precursors of non-transmissible chronic diseases. Physical inactivity and prolonged exposure to sedentary behaviours should be evaluated distinctly, considering the theoretical bases of each construct. The prolonged effect of sedentary behaviours and physical inactivity throughout lifespan enables the possibility of the harmful effects of such behaviours in old age and premature mortality.

Keywords: physical activity, sedentary lifestyle, review literature.

Artigo recebido a 03.11.2013; Aceite14.05.2014

${ }^{1}$ Universidade Federal do Triângulo Mineiro, Brasil

${ }^{2}$ Universidade do Estado da Bahia, campus Teixeira de Freitas, Brasil

* Autor correspondente: Núcleo de Estudos em Atividade Física \& Saúde. Universidade Federal do Triângulo Mineiro, Av. Tutunas, n 490, Tutunas, CEP: 38061-500, Uberaba, MG, Brasil;

E-mail: joilsonmeneguci@yahoo.com.br 


\section{INTRODUÇÃO}

A falta da prática regular de atividade física está associada à doença cardiovascular, à diabetes mellitus tipo 2, à obesidade, a alguns tipos de cancro e também à mortalidade por todas as causas (Hallal et al., 2012). Neste sentido, as evidências da relação inversa da atividade física com as doenças e agravos não transmissíveis colaboraram para a proposição de níveis mínimos de atividade física com o intuito de trazer benefícios para a saúde (R. Pate et al., 1995).

Até meados da década de 90 o modelo de promoção para a saúde visava a necessidade da mudança nos níveis de aptidão física para o alcance de benefícios; após esse período a incorporação do estilo de vida ativo tornou-se suficiente para obter benefícios para a saúde, independentemente da alteração no nível de aptidão física (Farias Júnior, 2011). Entretanto, com o avanço tecnológico, a realização de tarefas, tanto as diárias como as laborais, passou a ser mais simples, o que reduziu o tempo e a intensidade da atividade física e aumentou o tempo exposto a comportamentos sedentários (Hamilton, Hamilton, \& Zderic, 2007; Owen, Healy, Matthews, \& Dunstan, 2010).

O estudo do comportamento sedentário tem sido nos últimos 10 anos reconhecido como uma questão de saúde pública (Hallal et al., 2012) e investigações colocam em evidência que este comportamento está relacionado com efeitos deletérios para saúde, sendo necessário transmitir esta mensagem à população (Hamilton, Healy, Dunstan, Zderic, \& Owen, 2008).

A comunidade científica tem destacado que novas investigações a respeito do comportamento sedentário em diferentes regiões, culturas e os respetivos fatores correlatos, tendo como referência amostras de base populacional, fortalecem o entendimento das implicações para a saúde, além de possibilitarem a elaboração de diretrizes (Bauman et al., 2011; Hamilton et al., 2008; World Health Organization, 2010).

Recentes estudos epidemiológicos têm demonstrado que demasiado tempo despendido em comportamento sedentário, além de estar associado a doenças cardiovasculares, obesidade, síndrome metabólica, diabetes mellitus (Hamilton et al., 2007), trombose venosa (Howard et al., 2013), pode ser considerado um fator de risco para todas as causas de mortalidade, independentemente do nível de atividade física (Katzmarzyk, Church, Craig, \& Bouchard, 2009; van der Ploeg, Chey, Korda, Banks, \& Bauman, 2012).

Apesar do aumento da relevância do estudo do comportamento sedentário, principalmente pela sua relação com a saúde, os métodos de avaliação e as discussões ainda são incipientes e marcadas muitas das vezes por incoerências na utilização da terminologia (Owen et al., 2010).

Neste sentido, o presente estudo teve como objetivos: a) rever os termos operacionais utilizados para definir o comportamento sedentário; b) descrever as implicações fisiológicas relacionadas com o comportamento sedentário; e, c) os principais métodos de avaliação do comportamento sedentário.

\section{MÉTODO}

O presente estudo é uma revisão realizada a partir do levantamento de artigos publicados em periódicos indexados que tiveram o objetivo de discutir a temática do comportamento sedentário em relação à sua definição, implicações fisiológicas e métodos avaliativos.

Para o levantamento dos artigos na literatura realizou-se uma busca avançada nas bases de dados da Biblioteca Virtual em Saúde (BVS), National Library of Medicine (PubMed) e $W e b$ of Science. Utilizou-se a combinação do termo "sedentary behavior" com "definition", "physiological mechanisms" e "assessment methods", conforme apresentado na Figura 1.

Através do procedimento de busca adotado, selecionaram-se os artigos que apresentaram o conceito de comportamento sedentário, as implicações fisiológicas advindas deste comportamento e os métodos de avaliação. Também foi realizada uma busca manual a partir da leitura das referências dos artigos selecionados 
nas bases de dados pesquisadas, sendo que aqueles considerados relevantes para a discus- são e o entendimento dos constructos analisados foram incluídos neste artigo.

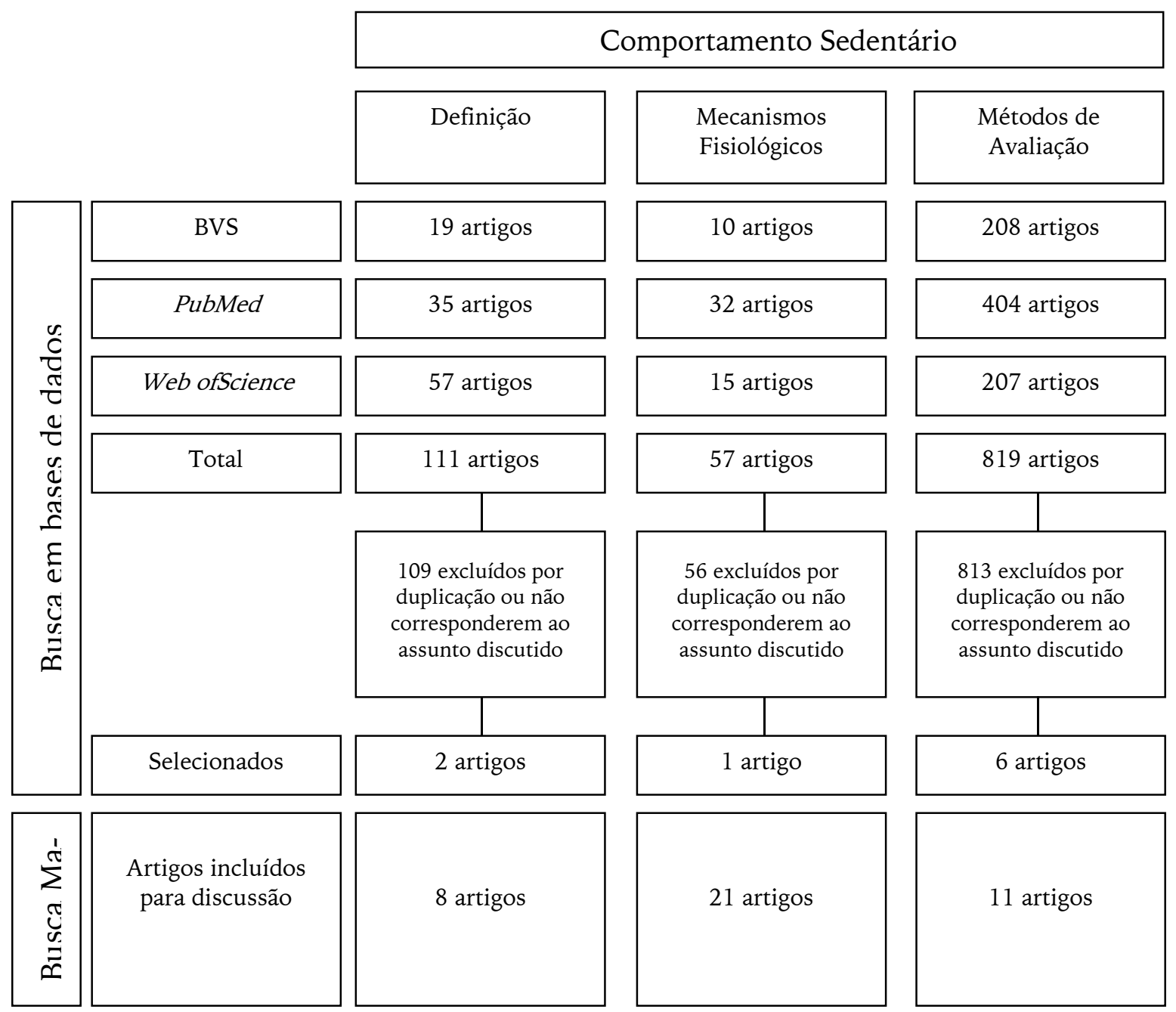

Figura 1. Organograma do processo de busca dos artigos

Relativamente à definição operacional, selecionaram-se, a partir da busca realizada, os trabalhos de Owen et al. (2010) e Pate, O'Neill, e Lobelo (2008). Adicionalmente foram incluídos trabalhos que discutiram a respeito do compêndio de atividade física (Ainsworth et al., 2000), acerca da topografia corporal (Hamilton et al., 2007, 2008) e do sono (Must \& Parisi, 2009; Patel \& Hu, 2008; Taheri, Lin, Austin, Young, \& Mignot, 2004). A diferenciação da nomenclatura comportamento sedentário de atividade física teve como base recomendações de intensidade, frequência e duração da prática de atividade física (R. Pate et al., 1995; World Health Organization, 2010).

Para discussão das implicações fisiológicas foram utilizados estudos que relacionaram o comportamento sedentário à mortalidade (Katzmarzyk et al., 2009; van der Ploeg et al., 2012) e à redução da expectativa de vida (Katzmarzyk \& Lee, 2012). De acordo com a busca realizada, o trabalho de Charansonney (2011) foi selecionado como referência para descrever os mecanismos fisiológicos do comportamento sedentário.

Em relação aos métodos de avaliação do comportamento sedentário, foram selecionados 
os artigos que discutiram tanto dispositivos tecnológicos (Grant, Ryan, Tigbe, \& Granat, 2006; Kerr et al., 2013; Ryde, Gilson, Suppini, \& Brown, 2012) como de autorrelato (Clark et al., 2011; A. L. Marshall, Miller, Burton, \& Brown, 2010; Rosenberg, Bull, Marshall, Sallis, \& Bauman, 2008). Como forma de demonstrar os métodos de avaliação do comportamento sedentário, adotou-se a estratégia de incluir os métodos de avaliação da atividade física (Florindo, Latorre, Jaime, Tanaka, \& Zerbini, 2004; Grant et al., 2006; Kriska \& Caspersen, 1997; Oliveira \& Maia, 2001; Rodrigo Siqueira Reis, Petroski, \& Lopes, 2000).

Com o intuito de atender o objetivo do estudo foi construído um modelo teórico para definir o comportamento sedentário, apresentar as implicações fisiológicas advindas deste comportamento e os métodos de avaliação.

\section{Comportamento sedentário: definição opera- cional}

Com o objetivo de transmitir uma mensagem à população mostrando que para alcançar benefícios para a saúde não é necessário participar em programas vigorosos de atividade física, as orientações para a prática com intensidade moderada passam a ser recomendadas na prevenção de doenças (R. Pate et al., 1995; World Health Organization, 2010).

Nos últimos anos tem sido observado um maior cuidado com o uso de denominações apropriadas na estratificação dos níveis de atividade física e comportamentos sedentários. A inatividade física vem sendo entendida como a condição de não atingir as diretrizes de saúde pública para os níveis recomendados de atividade física de intensidade moderada a vigorosa (AFMV) (Hallal et al., 2012). O comportamento sedentário tem sido definido para se referir à exposição a atividades com baixo dispêndio energético, atividades $\leq 1.5$ equivalentes metabólicos (METs) (Owen et al., 2010; R. R. Pate et al., 2008).
Apesar da evolução da área de atividade física e saúde no uso adequado de terminologias, ainda é possível encontrar na literatura o termo sedentário como sendo utilizado para descrever de forma inapropriada o baixo dispêndio energético em AFMV (Mullen et al., 2011). Esta falta de consenso na utilização das terminologias para evidenciar o nível indesejado de atividade física pode ocasionar uma interpretação ambígua na generalização dos resultados de estudos (Farias Júnior, 2011).

Frente à exposição dos diferentes termos utilizados, os constructos distintos dos comportamentos sedentário e atividade física são apresentados em organograma no intuito de exemplificar e padronizar a utilização das referidas terminologias (Figura 2).

Comportamento sedentário é o termo direcionado para as atividades que são realizadas na posição deitada ou sentada e que não aumentam o dispêndio energético acima dos níveis de repouso (Ainsworth et al., 2000; R. R. Pate et al., 2008). São exemplos de atividades sedentárias as que estão relacionadas com uma exigência energética baixa, como ver televisão, o uso do computador, assistir às aulas, trabalhar ou estudar numa mesa e a prática de jogos eletrónicos na posição sentada (Amorim \& Faria, 2012; Owen et al., 2010).

A simples posição em pé, mesmo sem a realização de alguma atividade, não é considerada como comportamento sedentário, podendo ser diferenciada das atividades sentadas, já que exige contração isométrica da musculatura para se opor à gravidade (Hamilton et al., 2007, 2008).

$\mathrm{Na}$ classificação do comportamento sedentário tanto a topografia corporal como o equivalente metabólico podem conduzir a dúvidas na interpretação no momento de estratificação do risco para a saúde, a exemplo das atividades de escrever ou digitar na posição sentada que correspondem a 1.8 METs, sendo este score metabólico similar à atividade de leitura na posição ortostática (Ainsworth et al., 2000). 


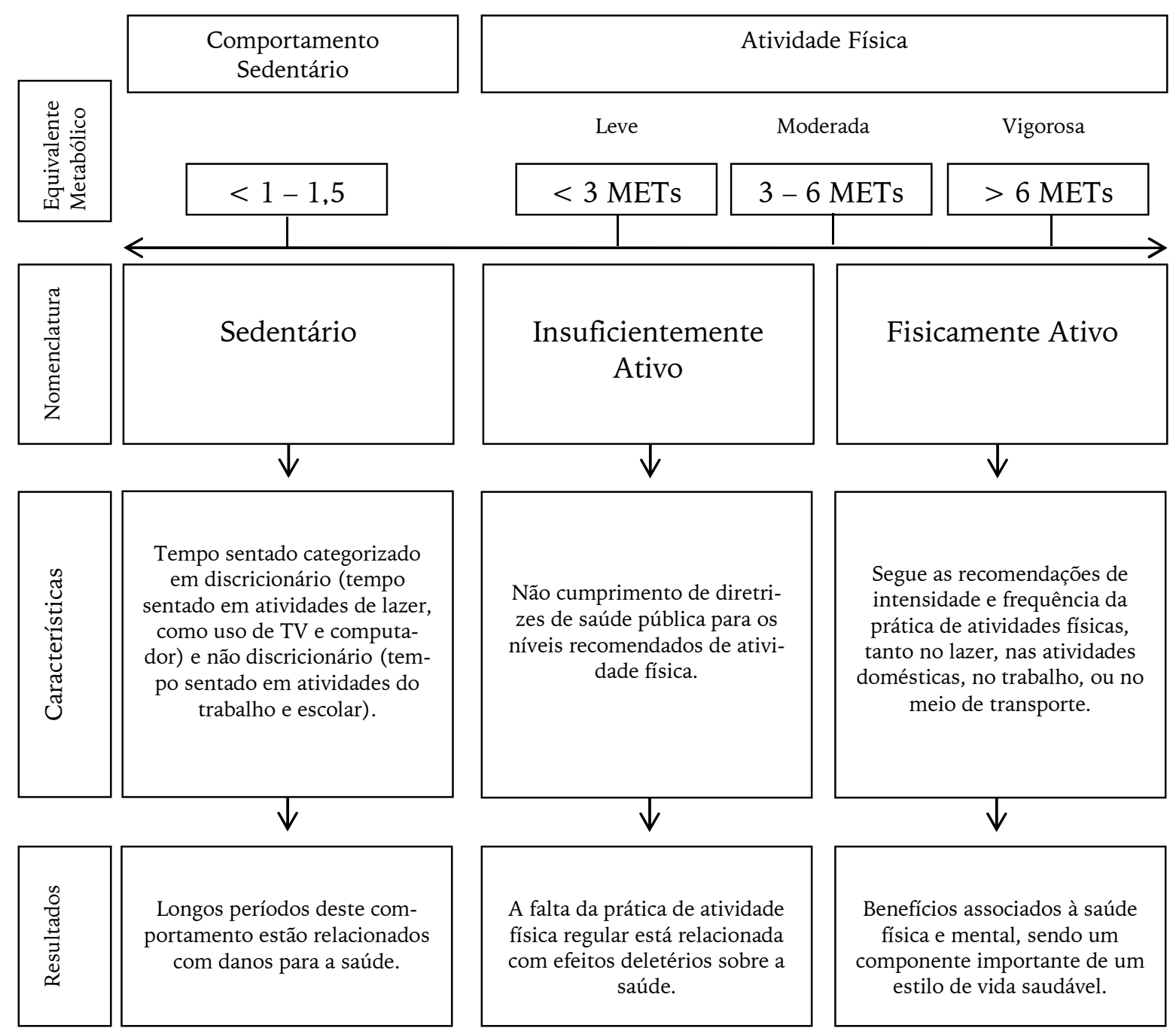

Figura 2 Organograma e definição operacional para os construtos comportamento sedentário e atividade física

Tendo como base a definição de comportamento sedentário, o sono é considerado uma atividade sedentária, pois o seu gasto energético é de .9 METs (Ainsworth et al., 2000). O tempo de sono recomendado para adultos é de 7 a 9 horas (National Sleep Foundation) a cada 24 horas, devido à necessidade orgânica de recuperação, portanto este período não deve ser quantificado como comportamento sedentário para estratificação de risco para a saúde (Owen et al., 2010).

Períodos de sono inferiores ou superiores aos recomendados para a saúde devem ser analisados na quantificação dos comportamentos sedentários de risco. A princípio podia-se esperar que a duração do sono curto, ou o "dé- bito do sono", estaria associada a maiores gastos diários de energia e, portanto, menor peso (Must \& Parisi, 2009). Entretanto, evidências clínicas e populacionais revelaram que a duração do sono curto está associada com o excesso de peso em adultos (Patel \& Hu, 2008).

A regulação do sono contribui para a manutenção do peso corporal e do metabolismo orgânico saudável (Must \& Parisi, 2009; Patel $\& \mathrm{Hu}, 2008$; Taheri et al., 2004). Num estudo de base populacional denominada de Wisconsin Sleep Cohort Study foram analisados distúrbios do sono de 1.024 voluntários, sendo identificado que os participantes com deficit de sono apresentavam alterações nos hormônios reguladores do apetite com a redução da greli- 
na e a elevação da leptina, e consequente aumento do índice de massa corporal (Taheri et al., 2004).

A relação do deficit de sono e o aumento do peso corporal envolvem efeitos metabólicos diretos, bem como de vias comportamentais indiretas, incluindo a presença de televisores e de meios de comunicação eletrónicos (Must \& Parisi, 2009). Nas sociedades ocidentais, onde a restrição crónica do sono é comum e os alimentos altamente calóricos estão amplamente disponíveis, as alterações nas concentrações dos hormônios reguladores do apetite com a redução do sono pode contribuir para a obesidade (Taheri et al., 2004).

\section{Comportamento sedentário: implicações fisi- ológicas}

Há consenso entre estudos para a condição elevada do tempo exposto a comportamentos sedentários estar associada a um maior risco de mortalidade (Katzmarzyk et al., 2009; van der Ploeg et al., 2012). Ou seja, por mais que o indivíduo seja ativo fisicamente, tal comportamento pode não compensar os efeitos adversos do tempo prolongado na posição sentada.

Com o objetivo de determinar a relação entre tempo sentado, doenças cardiovasculares, cancro e mortalidade por todas as causas, Katzmarzyk, Church, Craig, e Bouchard (2009) avaliaram o tempo sentado referido por 17.013 canadenses durante o período de 13 anos. Foi encontrado que o tempo sentado por um período prolongado estava associado positivamente com as doenças cardiovasculares e com a elevação das taxas de mortalidade por todas as causas.

Nesta mesma linha, analisando o tempo sentado de 222.497 australianos com 45 anos de idade, van der Ploeg, Chey, Korda, e Bau- man (2012) observaram que os indivíduos com maior tempo sentado apresentaram um risco relativo de mortalidade de 1.11 (95\%, IC: 1.08 $-1.15)$.

Em um recente estudo de meta análise desenvolvido com o objetivo de verificar os efeitos do comportamento sedentário na expectativa de vida da população dos Estados Unidos, foi identificado um aumento de dois anos na expectativa de vida com a redução do tempo diário despendido na posição sentada para menor que três horas e um aumento de 1.38 anos a partir da redução para menor de duas horas/dia de visualização de TV (Katzmarzyk \& Lee, 2012).

Os mecanismos pelos quais o comportamento sedentário aumenta o risco de mortalidade e doenças crónicas e suas consequências constituem a síndrome do comportamento sedentário. Os mecanismos explicativos para esta síndrome partem da premissa de que a imobilização proporciona o disparo de respostas estressoras responsáveis por efeitos deletérios para a saúde (Charansonney, 2011; Charansonney \& Després, 2010).

A acumulação de efeitos nocivos resultantes do longo tempo exposto a comportamentos sedentários ao longo do curso da vida poderá favorecer o desencadeamento ou a exacerbação de doenças crónicas na velhice e a mortalidade precoce (Charansonney, 2011).

O modelo exposto na Figura 3 explica como o comportamento sedentário pode aumentar o risco para doenças crónicas e eventos agudos, e como as pausas entre as atividades sedentárias podem diminuir estas consequências. Este modelo foi adaptado de Charansonney (2011) e complementado com os resultados de estudos descritos na sequência deste tópico. 


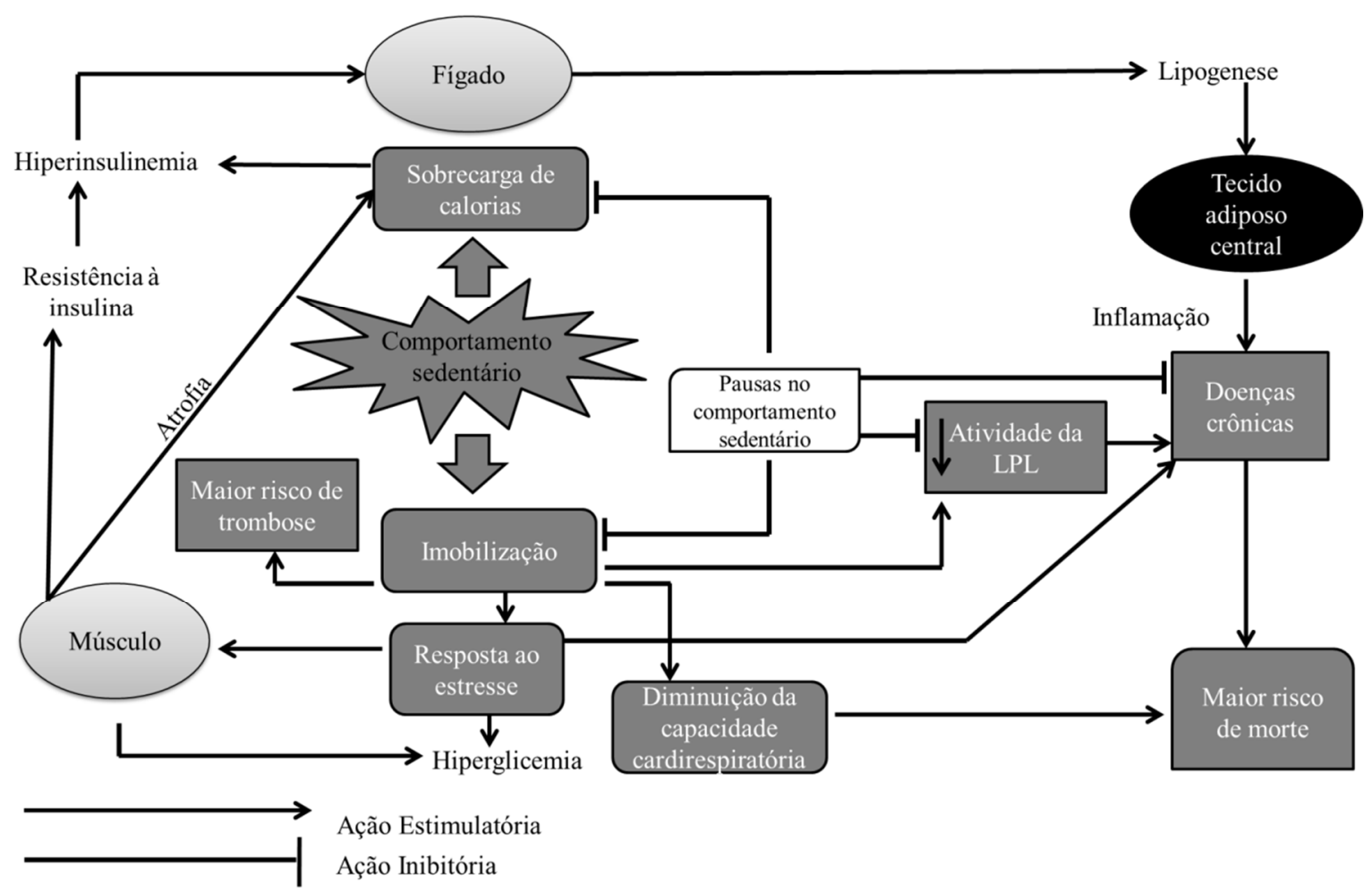

Figura 3. Comportamento sedentário e seus efeitos deletérios à saúde, adaptado de Charansonney (2011).

A imobilização é considerada um mecanismo estressor, o qual diminui a utilização de glicose pelos músculos, aumentando a resistência à insulina e ocasionando a atrofia muscular e a diminuição da utilização de energia pelos músculos inativos (Charansonney, 2011; Charansonney \& Després, 2010; Zhang, Chen, \& Fan, 2007). A energia é realocada para o fígado, o qual aumenta a produção de lipídios, que preferencialmente são armazenados no tecido adiposo da região central do corpo (Charansonney, 2011). Estes adipócitos tornam-se metabolicamente ativos quando carregados de gordura, e ambos produzem moléculas inflamatórias concomitantes à redução da secreção de adiponectinas anti-inflamatórias (Elks \& Francis, 2010).

Além disso, ocorre o aumento do número de macrófagos ativos que produzem citocinas pró-inflamatórias, que por sua vez desempenham um papel importante na patogénese das dislipidemias, hipertensão arterial e doenças cardíacas (Heber, 2010; Rasouli \& Kern, 2008). O estado inflamatório crónico pode representar um fator desencadeador da síndrome metabólica e ser responsável por disfunções endoteliais como a aterosclerose (Heber, 2010).

O aumento da ingestão calórica é outro fator que está associado a prejuízos para a saúde, proporcionando o aumento do acúmulo de gordura no fígado e nos adipócitos, desencadeando os efeitos deletérios para a saúde (Charansonney, 2011; Charansonney \& Després, 2010).

Por sua vez, o acúmulo de gordura corporal proporciona ao indivíduo dificuldade para realizar atividades aeróbias (Hunter, Weinsier, Zuckerman, \& Darnell, 2004) e consequentemente a redução do consumo máximo de oxigénio e o aumento do risco de morte por todas as causas (Kodama et al., 2009). O decréscimo da capacidade cardiorrespiratória pode ser o resultado do aumento do tempo exposto a comportamentos sedentários (Charansonney, 2011).

Estudos têm demonstrado que não basta medir o tempo total exposto a comportamen- 
tos sedentários, mas também o padrão deste comportamento, por exemplo, a existência de interrupções e o tempo de intervalo dessas pausas (Cooper et al., 2012; Healy, Matthews, Dunstan, Winkler, \& Owen, 2011). Entretanto, pouco se sabe sobre o intervalo de tempo em que o comportamento sedentário deve ser interrompido (Rutten, Savelberg, Biddle, \& Kremers, 2013).

O elevado tempo despendido em atividades sedentárias está associado a uma maior circunferência da cintura (Cooper et al., 2012). Por outro lado, valores menores de circunferência da cintura foram diagnosticados para indivíduos com maior frequência em pausas no comportamento sedentário (Cooper et al., 2012; Healy et al., 2011). As interrupções no tempo sedentário também estão relacionadas com os benefícios nas concentrações de proteína c-reativa e de glicose plasmática de jejum (Healy et al., 2008).

Outra consequência advinda do comportamento sedentário é o aumento do risco de desenvolver trombose. Um estudo recente demonstrou que a interrupção do comportamento sedentário está relacionada com um menor aumento de fibrinogénio no plasma e com a redução de parâmetros de volume de sangue que influenciam a viscosidade do sangue, reduzindo o risco de trombose venosa (Howard et al., 2013).

Pausas no tempo prolongado de comportamento sedentário devem ser incentivadas às pessoas, pois há indicativos de que interrupções de pelo menos de um minuto (Healy et al., 2008) em prolongadas atividades sedentárias contribuem para a redução dos efeitos nocivos deste comportamento para o corpo.

A interrupção do tempo sentado com sessões curtas de caminhada de intensidade leve ou moderada, numa proporção de dois minutos por 20 minutos em atividades sedentárias, reduz a glicose pós-prandial e os níveis de insulina em adultos com sobrepeso ou obesidade, podendo melhorar o metabolismo da glicose e os seus efeitos deletérios sobre a saúde, como os processos inflamatórios e a função endotelial prejudicada que são responsáveis por reduzir a espessura da camada íntimamédia, que por sua vez aumentam o risco de complicações cardiovasculares (Dunstan et al., 2012).

Estudos com modelos animais procuraram investigar os mecanismos fisiológicos que desencadeiam os efeitos maléficos decorrentes do tempo demasiado exposto ao comportamento sedentário (Bey \& Hamilton, 2003). Tais estudos indicam que o musculosquelético é o principal local para a alocação dos triglicéridos e da glicose plasmática, sendo que a falta de contração muscular faz desencadear o aumento dos triglicéridos, da glicose plasmática e a redução da atividade da lipoproteína lípase (LPL).

A LPL é uma enzima que regula a absorção de triglicerídeos e a produção de proteínas de alta densidade no músculo-esquelético (HDL). Sendo assim, quando se reduz a atividade enzimática da LPL reduz-se também a concentração de HDL no sangue, fator prejudicial à saúde, sendo evidenciado que a redução parcial da função da LPL, devido a um polimorfismo específico, foi associada ao aumento de 5 vezes na razão de probabilidades de morte e de doenças cardíacas coronárias (Wittrup, TybjærgHansen, \& Nordestgaard, 1999).

A maior parte da atividade da LPL é controlada pela contração muscular, pelo que desta forma, aqueles que passam longos períodos expostos a comportamentos sedentários não estimulam adequadamente a atividade dessa enzima (Hamilton et al., 2008). O ato de sair da posição sentada para a posição em pé já seria capaz de ativar o funcionamento da enzima LPL, e consequentemente evitar efeitos prejudiciais ao metabolismo dos lipídeos na produção de HDL (Bey \& Hamilton, 2003; Hamilton et al., 2007, 2008).

Os efeitos benéficos da pausa no tempo de comportamento sedentário também podem estar associados ao dispêndio energético. Aqueles indivíduos que realizam maior quantidade de pausas em atividades sedentárias apresentam maior gasto energético total em comparação com aqueles que não realizam pausas, 
contribuindo para um menor ganho de gordura corporal e um maior número de contrações musculares, que por sua vez estarão associadas ao menor risco de desenvolver alterações prejudiciais em marcadores metabólicos (Healy et al., 2008; Levine, 2004).

\section{Comportamento sedentário: procedimentos de avaliação}

O comportamento sedentário e a inatividade física não são sinónimos, pois ambos apresentam respostas fisiológicas diferentes em relação à saúde, portanto não podem ser mensurados e interpretados de maneira igual (R. R. Pate et al., 2008).

Métodos de avaliação da atividade física apresentam-se referenciados na literatura (Kriska \& Caspersen, 1997; Reis et al., 2000) e, entretanto, é necessária a sistematização de métodos de avaliação do comportamento sedentário.

Os instrumentos de medidas podem ser classificados sob dois parâmetros: aqueles que utilizam informações fornecidas pelos sujeitos (questionários, entrevistas e diários) e os que utilizam marcadores fisiológicos ou sensores de movimento para a avaliação direta de atividades em determinados períodos de tempo (Reis et al., 2000).

Outra classificação acerca dos métodos de avaliação da atividade física propõe a divisão em métodos laboratoriais (fisiológicos - água duplamente marcada, calorimetria direta e indireta; biomecânicos - plataforma de força) e métodos de terreno (diário; classificação profissional; questionários e entrevistas; observações comportamentais; monitorização mecânica e eletrónica - pedómetro, acelerómetro e monitor de frequência cardíaca) (Oliveira \& Maia, 2001).

Os métodos que utilizam informações fornecidas pelos sujeitos, conhecidos como de levantamento ou survey, são os métodos mais utilizados em pesquisas epidemiológicas; contudo, as medidas baseadas em informações fornecidas pelos sujeitos apresentam precisão limitada (Reis et al., 2000).
O uso de métodos que utilizam sensores de movimento, como pedómetros, acelerómetros, monitores tridimensionais de atividade e LSI's (Large-scale Integrators), são cada vez mais utilizados em estudos epidemiológicos (Oliveira \& Maia, 2001).

$\mathrm{O}$ avanço das tecnologias tem permitido o desenvolvimento de instrumentos pequenos e leves que permitem o armazenamento de dados por um determinado tempo. Tais instrumentos (sensores) são fixados no punho ou na região do quadril e medem o registo das acelerações do corpo ao longo do tempo, e a partir de equações de estimativa permitem o cálculo do dispêndio energético (Schoeller \& Racette, 1990). Por outro lado, os sensores de movimento apresentam algumas limitações quanto ao tipo de atividade e intensidade do movimento (Reis, 2003).

Em contrapartida, os métodos de avaliação do comportamento sedentário ainda estão em fase incipiente de desenvolvimento. A busca por métodos mais precisos para avaliar o comportamento sedentário parece estar entre as questões estratégicas das pesquisas na área de atividade física e saúde nos próximos anos (Marshall \& Merchant, 2013).

$\mathrm{Na}$ avaliação do tempo exposto a comportamentos sedentários devem-se distinguir as atividades realizadas no fim de semana das atividades dos dias úteis de semana, assim como deve ser considerado o tempo despendido em comportamento sedentário em diferentes domínios (por exemplo: trabalho, lazer, doméstico ou transporte) e ainda as interrupções ocorridas durante esse comportamento (Clark et al., 2011; Marshall et al., 2010).

O tempo sentado tem sido um dos marcadores específicos de comportamento sedentário utilizado como estratégia de avaliação. De acordo com o estudo de Rosenberg et al. (2008) o Questionário Internacional de Atividade Física (IPAQ) apresenta boa confiabilidade (.82) para o tempo sentado total (tempo sentado dia semana mais tempo sentado final de semana) e validade aceitável (.33) em relação ao acelerómetro. 
O constructo comportamento sedentário abrange os conceitos da topografia comportamental e da taxa metabólica. A topografia comportamental refere-se à forma do comportamento físico ou a relação entre as partes do corpo com o espaço (Marshall \& Merchant, 2013). Na pesquisa de comportamento sedentário, topografia é tipicamente operacionalizada como alocação de postura: deitado, reclinado, sentado e em pé (Marshall \& Merchant, 2013).

Mesmo comportamentos topográficos posturais iguais podem apresentar classificações de dispêndio energético diferentes, a exemplo do tempo sentado vendo TV versus o tempo sentado no trabalho (Ainsworth et al., 2000).

Quando o comportamento sedentário é definido pelo dispêndio de energia (por exemplo, o tempo despendido abaixo de 100 contagens por minuto de um acelerómetro), a postura é muitas vezes assumida como estando sentado ou deitado, fato que faz exacerbar os erros de medição nas estimativas do tempo de exposição aos comportamentos sedentários (Marshall \& Merchant, 2013).

As confusões dos termos operacionais acerca do comportamento sedentário diminuem o poder da validade externa dos estudos. É comum encontrar estudos que consideram os participantes sedentários porque não são fisicamente ativos, enquanto outros classificam os participantes sedentários por estarem envolvidos em atividades de baixo dispêndio de energia. A falta de padronização de instrumentos para avaliação do comportamento sedentário dificulta a comparação de dados tanto em regiões próximas como entre países (Bauman et al., 2011).

Dentre os instrumentos de avaliação do comportamento sedentário, os dispositivos de acelerometria triaxiais têm demonstrado bons índices psicométricos, podendo estes serem um recurso para futuras pesquisas epidemiológicas (Grant et al., 2006). Entretanto, os dispositivos via acelerometria não distinguem a posição do corpo na posição sentada da posição ortostática (Tremblay, Colley, Saunders, Healy, \& Owen, 2010). Porém, enquanto não se define um instrumento que atenda a todas as características desejadas, a combinação de instrumentos parece ser uma alternativa viável para fornecer dados mais precisos e confiáveis (Marshall \& Merchant, 2013).

O instrumento desenvolvido pela Microsoft denominado de SenseCam é um exemplo de instrumento com potencial para ser utilizado juntamente com outras estratégias para a avaliação do comportamento sedentário, a exemplo da acelerometria. O dispositivo de informação contextual, por meio de registo de imagens, o SenseCam passa a ser ativado por meio de sensor de acordo com a alteração de movimento, luz, temperatura ou presença de pessoas (Kerr et al., 2013).

Outro instrumento que foi desenvolvido para a avaliação do tempo sentado é o sitting pad (Ryde et al., 2012). O sitting pad é uma almofada que é instalada no assento de uma cadeira e contém um dispositivo para o registo do tempo em que a pessoa fica sentada. Esse dispositivo pode ser também programado para soar um alarme, alertando para o indivíduo interromper o seu tempo sentado.

A utilização de questionários em função da viabilidade em estudos populacionais tem sido uma estratégia bem pronunciada na avaliação do comportamento sedentário. Estudos têm utilizado questionários para a avaliação do tempo gasto sentado (Bauman et al., 2011) e de indicadores do comportamento sedentário, a exemplo do tempo de TV (Legnani et al., 2012).

O modelo da Figura 4 exemplifica os instrumentos de avaliação da atividade física e do comportamento sedentário, baseados em estudos que abordam as medidas de tais comportamentos (Florindo et al., 2004; Grant et al., 2006; Oliveira \& Maia, 2001; Reis et al., 2000). 


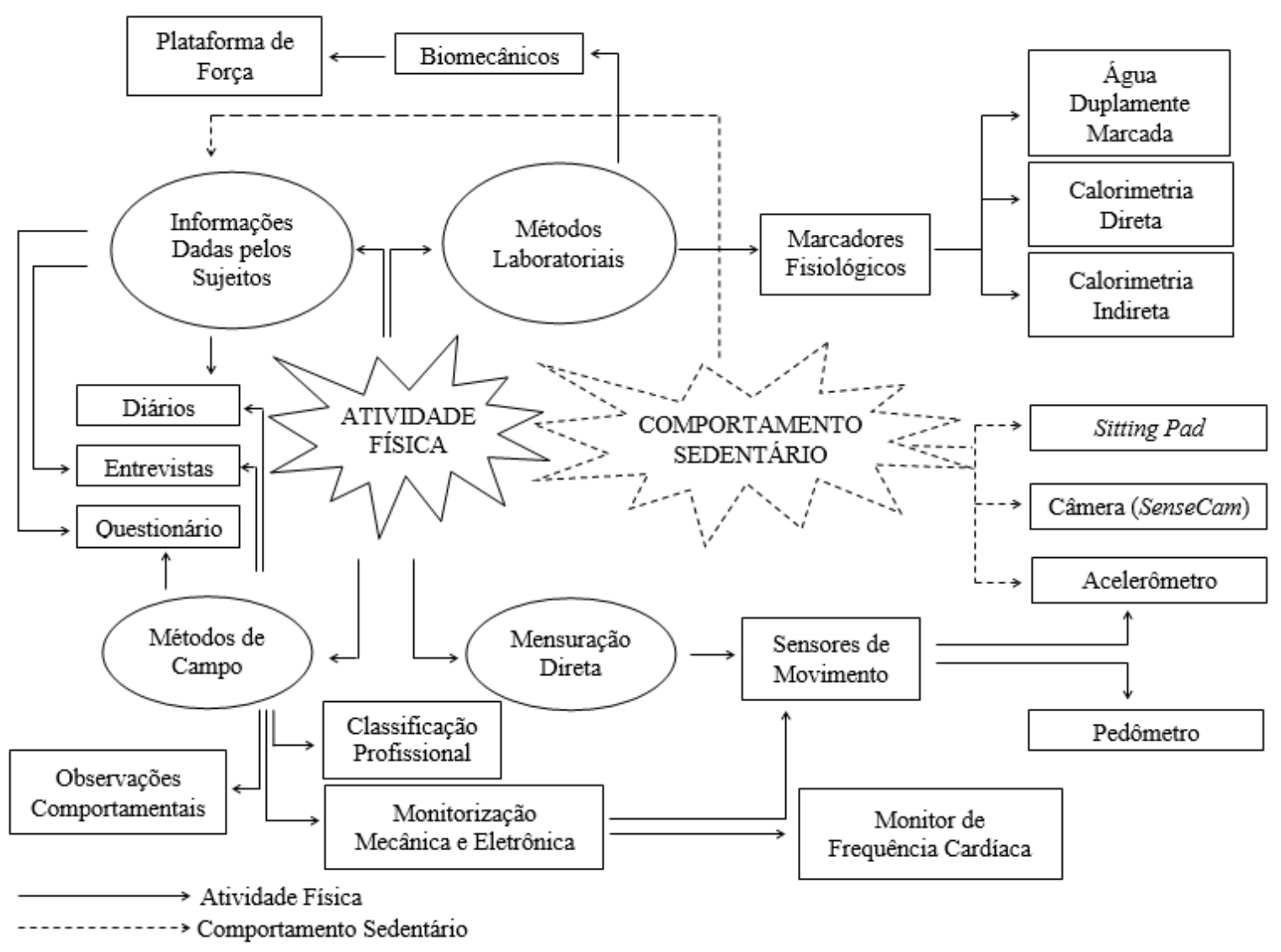

Figura 4. Instrumentos de avaliação da atividade física e comportamento sedentário

A utilização de instrumentos de auto relato tem sido uma estratégia adotada em estudos epidemiológicos no campo da atividade física e saúde, por serem de baixo custo e de fácil acesso (Florindo et al., 2004). Porém, algumas questões metodológicas devem ser consideradas, por exemplo, as características da população, o tempo do estudo realizado, o instrumento e as atividades desenvolvidas, já que estas peculiaridades podem limitar a precisão das estimativas obtidas (Kriska \& Caspersen, 1997).

\section{CONCLUSÕES}

Em resposta aos objetivos traçados inicialmente e com base na discussão apresentada, consideramos que o termo sedentário não deve ser utilizado para caracterizar aqueles indivíduos que não cumprem recomendações de prática de atividade física e saúde, esperando assim que pesquisas nesta área utilizem o ter- mo insuficientemente ativo para caracterizar aquelas pessoas que não cumprirem tais recomendações. Tanto a elevada exposição ao comportamento sedentário como à inatividade física estão relacionados com efeitos deletérios para a saúde. Estas duas variáveis comportamentais representam constructos independentes, devendo, portanto, serem avaliadas separadamente, tendo como pressuposto instrumentos com adequados índices psicométricos e que atendam o objetivo proposto ao estudo que se pretende desenvolver.

Nas últimas décadas tem sido crescente o tempo exposto a comportamentos sedentários independentemente da faixa etária. São necessárias estratégias mais adequadas para a monitorização deste comportamento, da mesma forma que são urgentes intervenções apropriadas que estimulem a adoção de estilos de vida ativos. Dentre as estratégias de fácil adoção e com potencial para minimizar os prejuízos para 
a saúde do tempo exposto a comportamentos sedentários preconizam-se as interrupções de curta duração entre períodos prolongados de tempo sentado.

Salientamos que a área do conhecimento denominada de epidemiologia da inatividade física ou do comportamento sedentário é relativamente recente, tendo em vista que o maior número de estudos concentra-se nas últimas três décadas. Há que considerar o fato da área ter surgido há relativamente pouco tempo, a necessidade da ampliação de estudos na tentativa de sanar lacunas e de avançar com o conhecimento a respeito do comportamento sedentário e da inatividade física.

\section{Agradecimentos:}

Nada a declarar.

\section{Conflito de Interesses:}

Nada a declarar.

\section{Financiamento:}

Nada a declarar.

\section{REFERÊNCIAS}

Ainsworth, B. E., Haskell, W. L., Whitt, M. C., Irwin, M. L., Swartz, A. M., Strath, S. J., ... Leon, A. S. (2000). Compendium of physical activities: an update of activity codes and MET intensities. Medicine and Science in Sports and Exercise, $32(9 \quad$ Suppl), S498-504. http://doi.org/10.1097/00005768-20000900100009

Amorim, P. R. S., \& Faria, F. R. (2012). Dispêndio energético das atividades humanas e sua repercussão para a saúde. Motricidade, 8(Supl. 2), S295-S302.

Bauman, A., Ainsworth, B. E., Sallis, J. F., Hagströmer, M., Craig, C. L., Bull, F. C., ... IPS Group. (2011). The descriptive epidemiology of sitting. A 20-country comparison using the International Physical Activity Questionnaire (IPAQ). American Journal of Preventive Medicine, 41(2), 228-235. http://doi.org/10.1016/j.amepre.2011.05.003

Bey, L., \& Hamilton, M. T. (2003). Suppression of skeletal muscle lipoprotein lipase activity dur- ing physical inactivity: a molecular reason to maintain daily low-intensity activity. The Journal of Physiology, 551(Pt 2), 673-682. http://doi.org/10.1113/jphysiol.2003.045591

Charansonney, O. L. (2011). Physical activity and aging: a life-long story. Discovery Medicine, 12(64), 177-185.

Charansonney, O. L., \& Després, J. P. (2010). Disease prevention-should we target obesity or sedentary lifestyle? Nature Reviews Cardiology, $\quad 7(8), \quad 468-472$. http://doi.org/10.1038/nrcardio.2010.68

Clark, B. K., Thorp, A. A., Winkler, E. A. H., Gardiner, P. A., Healy, G. N., Owen, N., \& Dunstan, D. W. (2011). Validity of self-reported measures of workplace sitting time and breaks in sitting time. Medicine and Science in Sports and Exercise, 43(10), 1907-1912. http://doi.org/10.1249/MSS.0b013e31821820 a2

Cooper, A. R., Sebire, S., Montgomery, A. A., Peters, T. J., Sharp, D. J., Jackson, N., ... Andrews, R. C. (2012). Sedentary time, breaks in sedentary time and metabolic variables in people with newly diagnosed type 2 diabetes. Diabetologia, 55(3), 589-599. http://doi.org/10.1007/s00125-011-2408-x

Dunstan, D. W., Kingwell, B. A., Larsen, R., Healy, G. N., Cerin, E., Hamilton, M. T., ... Owen, N. (2012). Breaking up prolonged sitting reduces postprandial glucose and insulin responses. Diabetes Care, 35(5), 976-983. http://doi.org/10.2337/dc11-1931

Elks, C. M., \& Francis, J. (2010). Central adiposity, systemic inflammation, and the metabolic syndrome. Current Hypertension Reports, 12(2), 99-104. http://doi.org/10.1007/s11906-0100096-4

Farias Júnior, J. C. (2011). (In)Atividade física e comportamento sedentário: estamos caminhando para uma mudança de paradigma? Revista Brasileira de Atividade Física \& Saúde, 16(4), 279-280. http://doi.org/10.12820/rbafs.v.16n4p279-280

Florindo, A. A., Latorre, M. do R. D. de O., Jaime, P. C., Tanaka, T., \& Zerbini, C. A. de F. (2004). Methodology to evaluation the habitual physical activity in men aged 50 years or more. $R e$ vista de Saúde Pública, 38(2), 307-314. http://doi.org/10.1590/S003489102004000200022 
Grant, P. M., Ryan, C. G., Tigbe, W. W., \& Granat, M. H. (2006). The validation of a novel activity monitor in the measurement of posture and motion during everyday activities. British Journal of Sports Medicine, 40(12), 992-997. http://doi.org/10.1136/bjsm.2006.030262

Hallal, P. C., Bauman, A. E., Heath, G. W., Kohl, H. W., Lee, I.-M., \& Pratt, M. (2012). Physical activity: more of the same is not enough. Lancet, 380(9838), 190-191. http://doi.org/10.1016/S01406736(12)61027-7

Hamilton, M. T., Hamilton, D. G., \& Zderic, T. W. (2007). Role of low energy expenditure and sitting in obesity, metabolic syndrome, type 2 diabetes, and cardiovascular disease. Diabetes, 56(11), 2655-2667. http://doi.org/10.2337/db07-0882

Hamilton, M. T., Healy, G. N., Dunstan, D. W., Zderic, T. W., \& Owen, N. (2008). Too Little Exercise and Too Much Sitting: Inactivity Physiology and the Need for New Recommendations on Sedentary Behavior. Current Cardiovascular Risk Reports, 2(4), 292-298. http://doi.org/10.1007/s12170-008-0054-8

Healy, G. N., Dunstan, D. W., Salmon, J., Cerin, E., Shaw, J. E., Zimmet, P. Z., \& Owen, N. (2008). Breaks in sedentary time: beneficial associations with metabolic risk. Diabetes Care, 31(4), 661-666. http://doi.org/10.2337/dc072046

Healy, G. N., Matthews, C. E., Dunstan, D. W., Winkler, E. A. H., \& Owen, N. (2011). Sedentary time and cardio-metabolic biomarkers in US adults: NHANES 2003-06. European Heart Journal, 32(5), 590-597. http://doi.org/10.1093/eurheartj/ehq451

Heber, D. (2010). An integrative view of obesity. The American Journal of Clinical Nutrition, 91(1), 280S-283S. http://doi.org/10.3945/ajcn.2009.28473B

Howard, B. J., Fraser, S. F., Sethi, P., Cerin, E., Hamilton, M. T., Owen, N., ... Kingwell, B. A. (2013). Impact on Hemostatic Parameters of Interrupting Sitting with Intermittent Activity. Medicine and Science in Sports and Exercise, 45(7), 1285-1291. http://doi.org/10.1249/MSS.0b013e318285f57 e

Hunter, G. R., Weinsier, R. L., Zuckerman, P. A., \& Darnell, B. E. (2004). Aerobic fitness, physiologic difficulty and physical activity in Black and White women. International Journal of Obesity, 28(9), 1111-1117. http://doi.org/10.1038/sj.ijo.0802724

Katzmarzyk, P. T., Church, T. S., Craig, C. L., \& Bouchard, C. (2009). Sitting Time and Mortality from All Causes, Cardiovascular Disease, and Cancer. Medicine and Science in Sports and Exercise, 41(5), 998-1005. http://doi.org/10.1249/MSS.0b013e31819303 55

Katzmarzyk, P. T., \& Lee, I.-M. (2012). Sedentary behaviour and life expectancy in the USA: a cause-deleted life table analysis. Bmj Open, 2(4), e000828. http://doi.org/10.1136/bmjopen-2012-000828

Kerr, J., Marshall, S. J., Godbole, S., Chen, J., Legge, A., Doherty, A. R., ... Foster, C. (2013). Using the SenseCam to improve classi-fications of sedentary behavior in free-living settings. American Journal of Preventive Medicine, 44(3), 290-296. http://doi.org/10.1016/j.amepre.2012.11.004

Kodama, S., Saito, K., Tanaka, S., Maki, M., Yachi, Y., Asumi, M., ... Sone, H. (2009). Cardiorespiratory Fitness as a Quantitative Predictor of All-Cause Mortality and Cardiovascular Events in Healthy Men and Women A Metaanalysis. Jama-Journal of the American Medical Association, 301(19), 2024-2035. http://doi.org/10.1001/jama.2009.681

Kriska, A. M., \& Caspersen, C. J. (1997). Introduction to a collection of physical activity questionnaires. Medicine \& Science in Sports \& Exercise, 29(6), 5-9. http://doi.org/10.1097/00005768-19970600100003

Legnani, E., Legnani, R. F., Dellagrana, R. A., Silva, M. P., Barbosa Filho, V. C., \& Campos, W. (2012). Comportamentos de risco à saúde e excesso de peso corporal em escolares de Toledo, Paraná, Brasil. Motricidade, 8(3), 59-70. http://doi.org/10.6063/motricidade.8(3).1157

Levine, J. A. (2004). Nonexercise activity thermogenesis (NEAT): environment and biology. American Journal of Physiology-Endocrinology and Metabolism, 286(5), E675-E685. http://doi.org/10.1152/ajpendo.00562.2003

Marshall, A. L., Miller, Y. D., Burton, N. W., \& Brown, W. J. (2010). Measuring total and domain-specific sitting: a study of reliability and validity. Medicine and Science in Sports and Exercise, 42(6), 1094-1102. 
http://doi.org/10.1249/MSS.0b013e3181c5ec1 8

Marshall, S. J., \& Merchant, G. (2013). Advancing the Science of Sedentary Behavior Measurement. American Journal of Preventive Medicine, 44(2), 190-191. http://doi.org/10.1016/j.amepre.2012.11.001

Mullen, S. P., Olson, E. A., Phillips, S. M., Szabo, A. N., Wojcicki, T. R., Mailey, E. L., ... McAuley, E. (2011). Measuring enjoyment of physical activity in older adults: invariance of the physical activity enjoyment scale (paces) across groups and time. International Journal of Behavioral Nutrition and Physical Activity, 8, 103. http://doi.org/10.1186/1479-5868-8-103

Must, A., \& Parisi, S. M. (2009). Sedentary behavior and sleep: paradoxical effects in association with childhood obesity. International Journal of Obesity (2005), 33 Suppl 1, S82-86. http://doi.org/10.1038/ijo.2009.23

Oliveira, M. M., \& Maia, J. A. (2001). Avaliação da actividade física em contextos epidemiológicos. Uma revisão da validade e fiabilidade do acelerómetro Tritrac-R3D, do pedómetro Yamax Digi-Walker e do questionário de Baecke. Revista Portuguesa de Ciências do Desporto, 1(3), 73-88.

Owen, N., Healy, G. N., Matthews, C. E., \& Dunstan, D. W. (2010). Too much sitting: the population health science of sedentary behavior. Exercise and Sport Sciences Reviews, 38(3), 105-113.

http://doi.org/10.1097/JES.0b013e3181e373a 2

Patel, S. R., \& Hu, F. B. (2008). Short sleep duration and weight gain: a systematic review. Obesity (Silver Spring, Md.), 16(3), 643-653. http://doi.org/10.1038/oby.2007.118

Pate, R., Pratt, M., Blair, S., Haskell, W., Macera, C., Bouchard, C., ... Wilmore, J. (1995). PhysicalActivity and Public-Health - a Recommendation from the Centers-for-Disease-Controland-Prevention and the American-College-ofSports-Medicine. Jama-Journal of the American Medical Association, 273(5), 402-407. http://doi.org/10.1001/jama.273.5.402

Pate, R. R., O'Neill, J. R., \& Lobelo, F. (2008). The evolving definition of «sedentary». Exercise and Sport Sciences Reviews, 36(4), 173-178. http://doi.org/10.1097/JES.0b013e3181877d1 a
Rasouli, N., \& Kern, P. A. (2008). Adipocytokines and the metabolic complications of obesity. The Journal of Clinical Endocrinology and Metabolism, 93(11 Suppl 1), S64-S73. http://doi.org/10.1210/jc.2008-1613

Reis, R. S. (2003). Medidas de atividades física: métodos e instrumentos. Em M. V. G. de Barros \& M. V. Nahas (Eds.), Medidas da atividade física: teoria e aplicação em diversos grupos populacionais (pp. 29-42). Londrina: Midiograf.

Reis, R. S., Petroski, E. L., \& Lopes, A. da S. (2000). Medidas da atividade física: revisão de métodos. Revista Brasileira de Cineantropometria e Desempenho Humano, 2(1), 89-96.

Rosenberg, D. E., Bull, F. C., Marshall, A. L., Sallis, J. F., \& Bauman, A. E. (2008). Assessment of sedentary behavior with the International Physical Activity Questionnaire. Journal of Physical Activity \& Health, 5(Suppl 1), S3044.

Rutten, G. M., Savelberg, H. H., Biddle, S. J. H., \& Kremers, S. P. J. (2013). Interrupting long periods of sitting: good STUFF. The International Journal of Behavioral Nutrition and Physical Activity, 10, 1. http://doi.org/10.1186/14795868-10-1

Ryde, G. C., Gilson, N. D., Suppini, A., \& Brown, W. J. (2012). Validation of a novel, objective measure of occupational sitting. Journal of Occupational Health, 54(5), 383-386. http://doi.org/10.1539/joh.12-0091-BR

Schoeller, D. A., \& Racette, S. B. (1990). A review of field techniques for the assessment of energy expenditure. The Journal of Nutrition, 120 Suppl 11, 1492-1495.

Taheri, S., Lin, L., Austin, D., Young, T., \& Mignot, E. (2004). Short Sleep Duration Is Associated with Reduced Leptin, Elevated Ghrelin, and Increased Body Mass Index. PLoS Med, 1(3), e62.

http://doi.org/10.1371/journal.pmed.0010062

Tremblay, M. S., Colley, R. C., Saunders, T. J., Healy, G. N., \& Owen, N. (2010). Physiological and health implications of a sedentary lifestyle. Applied Physiology, Nutrition, and Metabolism, 35(6), 725-740. http://doi.org/10.1139/H10-079

Van der Ploeg, H. P., Chey, T., Korda, R. J., Banks, E., \& Bauman, A. (2012). Sitting time and allcause mortality risk in 222497 Australian adults. Archives of Internal Medicine, 172(6), 
174 | J Meneguci, DT Santos, RB Silva, RG Santos, JE Sasaki, S Tribess, R Damião, JV Júnior

494-500.

http://doi.org/10.1001/archinternmed.2011.2 174

Wittrup, H. H., Tybjærg-Hansen, A., \& Nordestgaard, B. G. (1999). Lipoprotein Lipase Mutations, Plasma Lipids and Lipoproteins, and Risk of Ischemic Heart Disease A MetaAnalysis. Circulation, 99(22), 2901-2907. http://doi.org/10.1161/01.CIR.99.22.2901
World Health Organization. (2010). Global recommendations on physical activity for health. Genebra: World Health Organization. Obtido de

http://whqlibdoc.who.int/publications/2010/9 789241599979_eng.pdf

Zhang, P., Chen, X., \& Fan, M. (2007). Signaling mechanisms involved in disuse muscle atrophy. Medical Hypotheses, 69(2), 310-321. http://doi.org/10.1016/j.mehy.2006.11.043 